\title{
Improvement of Acute Respiratory Distress Syndrome with Ethyl Alcohol Infusion into the Airway: A Case Report
}

Takahashi $\mathbf{G}^{1 *}$ and Endo $\mathrm{S}^{2}$

${ }^{1}$ Department of Critical Care, Disaster and General Medicine, School of Medicine, Iwate Medical University, Morioka, Japan

${ }^{2}$ Morioka Yuai Hospital, Morika, Japan

\begin{abstract}
Background:This case involves a male in his late thirties who was transported from another institution to our hospital in a state of haemorrhagic shock after receiving severe injuries in a traffic accident.

Case Presentation: After hospitalisation and artificial respiration under sedation, his respiratory condition worsened on the $2^{\text {nd }}$ hospital day, and he was diagnosed with acute respiratory distress syndrome (ARDS) with an arterial oxygen partial pressure to fractional inspired oxygen $\left[\mathrm{PaO}_{2} / \mathrm{FiO}_{2}(\mathrm{P} / \mathrm{F})\right]$ ratio of $\leq 300$. A large amount of bloody foamy sputum was expectorated on the $4^{\text {th }}$ hospital day, and his P/F ratio decreased to 44 ; the amount of sputum did not reduce even after implementing sputum suction every 3-5 min. We considered introducing an extracorporeal membrane oxygenation device, but because it was an emergency situation with $<80 \%$ of percutaneous oxygen saturation, we instead performed suction operation several times immediately after injecting $10 \mathrm{cc}$ of ethyl alcohol (76.9 to $81.6 \mathrm{vol} \%$ ) into the pulmonary alveoli. Subsequently, bloody foamy sputum expectoration was suddenly decreased, and the patient recovered from ARDS on the $7^{\text {th }}$ hospital day, with a marked improvement in his $\mathrm{P} / \mathrm{F}$ ratio.
\end{abstract}

Conclusions: We have reported the implications of our continuous measurement for neutrophil elastase, interleukin 8 and surfactant protein $\mathrm{D}$ in blood and sputum, as well as how these metrics related to the patient's respiratory state.

Keywords: Acute respiratory distress syndrome; Respiratory status; $\mathrm{PaO}_{2} / \mathrm{FiO}_{2}$ ratio

Abbreviations: ARDS: Acute Respiratory Distress Syndrome; ECMO: Extracorporeal Membrane Oxygenation; IL-8: Interleukin 8; $\mathrm{PaO}_{2} / \mathrm{FiO}_{2}(\mathrm{P} / \mathrm{F})$ ratio: Arterial Oxygen Partial Pressure to Fractional Inspired Oxygen Ratio; SP-D: Surfactant Protein D

\section{Background}

Acute respiratory distress syndrome (ARDS) can arise due to various causes and is often seen in patients with traumatic haemorrhagic shock [1]. The prognosis of patients with ARDS remains poor, and the respiratory management of these patients involves numerous difficulties. In ARDS cases, the activation of inflammatory cells, such as neutrophils, against invasion leads to vascular endothelial and alveolar epithelial cell injuries, which produces enhanced vascular permeability, thus resulting in pulmonary oedema.

We have previously reported the efficacy of alveolar lavage with ethyl alcohol in a case of post-multiple fracture ARDS encountered by us, which was difficult to treat, and also discussed its relationship with neutrophil elastase and interleukin 8 (IL-8). We recently encountered another case of ARDS in a similar trauma patient, wherein ethyl alcohol administration into the pulmonary alveoli dramatically improved the patient's respiratory status. The blood and sputum levels of neutrophil elastase, IL- 8 and surfactant protein D (SP-D), elaborated by type II alveolar epithelial cells [2], were consecutively measured to assess their relation to the respiratory status.

\section{Case Presentation}

The patient was a male in his late thirties with an unremarkable family history. At about 6 am, the patient was driving a heavy truck that happened to skid on a frozen road surface, and that track fell to a cliff beneath about 15 meters. The patient's legs were trapped beneath the dashboard of the truck, and he was rescued approximately $4 \mathrm{~h}$ later. He was rushed to a nearby hospital and then transferred to our hospital 3 $\mathrm{h}$ later (about $8 \mathrm{~h}$ after the injury).

On admission, the systolic pressure was about $80 \mathrm{~mm} \mathrm{Hg}$, clearly indicating haemorrhagic shock. While the patient was receiving an emergency blood transfusion, imaging examinations to assess the extent of injuries were performed. He was found to have a fracture of the pelvis, an open fracture of the right femur, right lower leg fractures, an open fracture of the left femur and open fractures of the left lower leg bones. No trauma was seen on the head, chest or abdomen.

The systolic pressure improved to $100 \mathrm{~mm} \mathrm{Hg}$ approximately $1 \mathrm{~h}$ after his arrival at our hospital. Fracture treatment was then initiated, and the patient was admitted to the intensive care unit. At that time, there was no clinical sign of concern regarding his respiratory status, and his arterial oxygen partial pressure to fractional inspired oxygen $\left[\mathrm{PaO}_{2} / \mathrm{FiO}_{2}(\mathrm{P} / \mathrm{F})\right]$ ratio was 322 (Figure $1 \mathrm{~A}$ ), while the systolic pressure was consistently maintained at $110-120 \mathrm{~mm} \mathrm{Hg}$. However, on the $2^{\text {nd }}$ hospital day, his breathing rapidly became laboured; he was intubated and mechanical ventilation was initiated since his $\mathrm{P} / \mathrm{F}$ ratio became $\leq$ 300. On echocardiography, there was no decline in the left ventricular ejection fraction or expansion of the left atrium; however, bilateral infiltration shadows were observed in his chest X-ray, which were diagnostic of ARDS (Figure 1B). A large amount of bloody foamy sputum was expectorated on the $4^{\text {th }}$ hospital day, and his $\mathrm{P} / \mathrm{F}$ ratio decreased to 44 . The amount of sputum was not reduced even after implementing sputum suction every 3-5 min along with corticosteroid administration and other measures such as modification of mechanical ventilation parameters. During the night of the $4^{\text {th }}$ hospital day, we

*Corresponding author: Gaku Takahashi, Division of Critical Care Medicine Department of Critical Care, Disaster, and General Medicne, School of Medicine, Iwate Medical University, 19-1 Uchimaru, Morioka 020-8505, Japan, Phone: +81 19-651-5111, Fax: +81-19-651-5151; E-mail: gakut@iwate-med.ac.jp

Received July 17, 2018; Accepted July 24, 2018; Published July 31, 2018

Citation: Takahashi G, Endo S (2018) Improvement of Acute Respiratory Distress Syndrome with Ethyl Alcohol Infusion into the Airway: A Case Report. J Pulm Respir Med 8: 464. doi: 10.4172/2161-105X.1000464

Copyright: $@ 2018$ Takahashi G, et al. This is an open-access article distributed under the terms of the Creative Commons Attribution License, which permits unrestricted use, distribution, and reproduction in any medium, provided the original author and source are credited. 
considered introducing an extracorporeal membrane oxygenation (ECMO) device because this was an emergency situation with $<80 \%$ of percutaneous oxygen saturation. However, since it takes about $2 \mathrm{~h}$ to implement such a step during the night shift, we conducted the suction procedure several times after injecting $10 \mathrm{cc}$ of ethyl alcohol (76.9 to $81.6 \mathrm{vol} \%$ ) into the pulmonary alveoli at once as an emergency measure against hypoxic encephalopathy (Figure 1C). Fortunately, this was followed by a dramatic decrease in the amount of bloody foamy sputum produced. The procedure was repeated once, approximately $10 \mathrm{~min}$ later. Following the second administration of ethyl alcohol, there was little or zero production of bloody foamy sputum, and percutaneous oxygen saturation improved to approximately 90; therefore, ethyl alcohol was not administered further. Bilateral infiltrative shadows in the chest X-ray markedly improved on the $6^{\text {th }}$ hospital day. The P/F ratio exceeded 300 and the patient recovered from ARDS on the $7^{\text {th }}$ hospital day (Figure 1D).

During the time when he manifested the signs of ARDS, the patient's serum neutrophil elastase level was $1770 \mathrm{ng} / \mathrm{mL}$, sputum neutrophil elastase level was $19700 \mathrm{ng} / \mathrm{mL}$, serum IL- 8 level was 1210 $\mathrm{pg} / \mathrm{mL}$ and serum SP-D level was $670 \mathrm{ng} / \mathrm{mL}$, that is, the levels of all parameters were worryingly elevated. Remarkably, in response to the treatment, his serum and sputum neutrophil elastase levels, as well as serum IL-8 and SP-D levels decreased almost simultaneously, along with a marked increase in the $\mathrm{P} / \mathrm{F}$ ratio (Figure 2).

The patient also received a one-stage open reduction for his numerous fractures. He was transferred to another hospital for rehabilitation at 3 weeks after this surgery, where he was under a rehabilitation programme for about 4 months; he was discharged home with practically no disability.

\section{Discussion}

For this case, the frequent suction of profuse amounts of bloody foamy sputum and modification of positive end-expiratory pressure (PEEP) (5 to $15 \mathrm{mmHg}$ ) and tidal volume (300 to $650 \mathrm{~mL}$ ) performed over approximately $2 \mathrm{~h}$ failed to reduce the tremendous amount of sputum discharge.

We administered ethyl alcohol in this situation in line with a report of improvement in oxygenation resulting from a reduction of surface tension within the alveoli by ethyl alcohol inhalation and also in the light of gratifying results obtained through intra-tracheal ethyl alcohol infusion in a case of ARDS similar to ours. The results produced in the case documented herein clearly confirm the reproducibility of the response observed in these earlier reports [2].

Although it is uncertain how deep the ethyl alcohol injected in the vicinity of a tip of an endotracheal tube can reach within the alveolar spaces of the lung, this procedure possibly exerted a definite effect as the bloody foamy sputum secretion rapidly diminished after ethyl alcohol administration.

In response to challenges such as traumas, inflammatory cytokines and many other mediators elaborated in the body [3], the stimulated neutrophils produce large amounts of proteolytic enzymes such as neutrophil elastase. These factors are considered to have a profound bearing on the pathophysiological state of progression into multiorgan failure, including ARDS [4,5].

Neutrophil elastase has an extremely potent proteolytic enzyme activity and can reduce all constituent proteins of the body. In our case, the serum neutrophil elastase level was markedly elevated when the patient had ARDS. The sputum neutrophil elastase levels were also found to be elevated and eventually changed in parallel with the blood (serum) neutrophil elastase levels over time. Nevertheless, the sputum neutrophil elastase levels were one order of magnitude higher than the blood neutrophil elastase levels, possibly implying that a large amount of neutrophil elastase is produced at the site of inflammation.

The serum IL-8 level, which has neutrophil-activating properties, also changed in parallel with the blood (serum) and sputum neutrophil elastase levels over time, suggesting the possible involvement of IL-8 in the production of neutrophil elastase, which is consistent with our finding in a previous study [4].

We have also reported the possible involvement of neutrophil elastase in the impairment of the pulmonary surfactant [6,7] which is synthesised in and secreted from alveolar type II epithelial cells that cover the inner surfaces of the alveoli to prevent end-expiratory alveolar collapse [5]. The inhibition of this pulmonary surfactant activity by proteins in various lung oedema fluids is believed to be involved in the development of respiratory failure in ARDS. Even in this extreme case, the inhibitory action of SP-D on the pulmonary surfactant showed a high value at the onset of ARDS, and the acuteness of the situation decreased with improvement in the patient's respiratory condition.

Although the mechanism of improvement of oxygenation capacity in this case is not clear, no reports have indicated that ethyl alcohol suppresses the production of IL-8 and neutrophil elastase. Therefore, there is little evidence to infer that decreasing these values will directly improve respiratory function.

In our case, as reported by Luisada et al. the surface tension of the moisture coating on the alveolar surface was directly reduced by ethyl alcohol injection with very low surface tension and alveolar collapse was improved, while the formation of foam was suppressed. As a result, the ejection of bloody foamy sputum decreased and oxygenation improved. Further, the production of IL-8 and neutrophil elastase decreased, and the damage to the pulmonary surfactant was also suppressed.

As an animal experiment, in 1993, Waugh reported the effect of inhalation of alcohol vapor using rabbits drowning models. Since the alveolar edema improved with the same mechanism as reported by Luisada et al., The oxygenation ability was significantly improved in the group inhaling alcohol vapor compared to the group in which water vapor was inhaled [8].

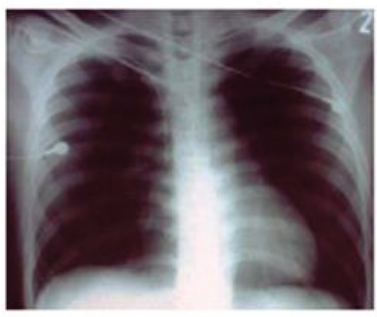

Figure 1A: On admission

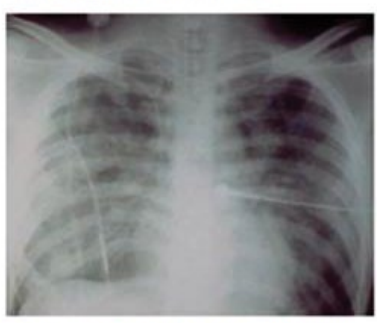

Figure 1C: On the 4th hospital day

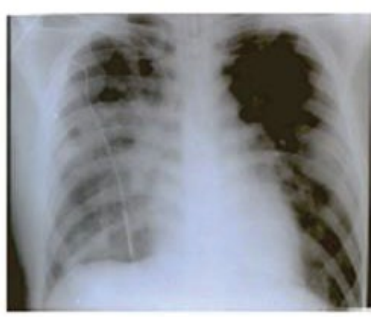

Figure 1B: On the 2nd hospital day

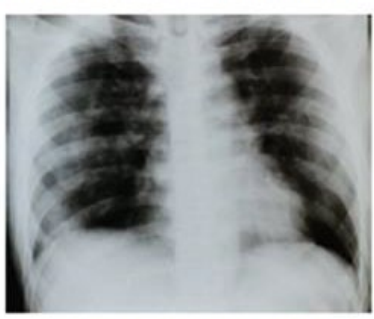

Figure 1D: On the 6th hospital day
Figure 1: Chest $\mathrm{X}$-ray in progress. 
Citation: Takahashi G, Endo S (2018) Improvement of Acute Respiratory Distress Syndrome with Ethyl Alcohol Infusion into the Airway: A Case Report. J Pulm Respir Med 8: 464. doi: 10.4172/2161-105X.1000464

Page 3 of 4

Even in the study we previously conducted, $200 \mu$ of distilled water was dropped on a waterproof surgical cloth to form a sphere, and when a small amount of ethyl alcohol was dropped on to it, the sphere immediately flattened and the surface tension decreased (Figure 3). It was also confirmed that the foam began disappearing immediately even when a small amount of ethyl alcohol was sprayed on a foam cleanser detergent, completely disappearing in about $10 \mathrm{~s}$ (Figure 4).

Since it is extremely uncommon to encounter a case like ours, it is difficult to accumulate relevant evidence. However, the gratifying outcomes obtained by us in two cases, including the present one, suggests the potential efficacy of ethyl alcohol infusion in such circumstances. Also, no adverse reactions were encountered. The optimal dose of ethyl alcohol, the optimal timing of its administration and the optimal method of its administration are yet to be fully explored.
Regarding the intra-tracheal injection of ethyl alcohol, considering the time required for introducing ECMO, it was decided that the risk of hypoxic encephalopathy would be increased; therefore, it was clearly explained to the family that it was an unauthorised method, and it was used only after obtaining consent.

As far as we could search, since the report of Boxman in 1958, we have not found a report used on the human body, and the safety and effectiveness are not sufficiently verified as a treatment method [9]. We never choose an evidence-free treatment. We would like the readers to understand that this case was an emergency situation.

\section{Conclusion}

By administration of ethyl alcohol into the pulmonary alveoli, the amount of bloody foamy sputum decreased markedly, and after that

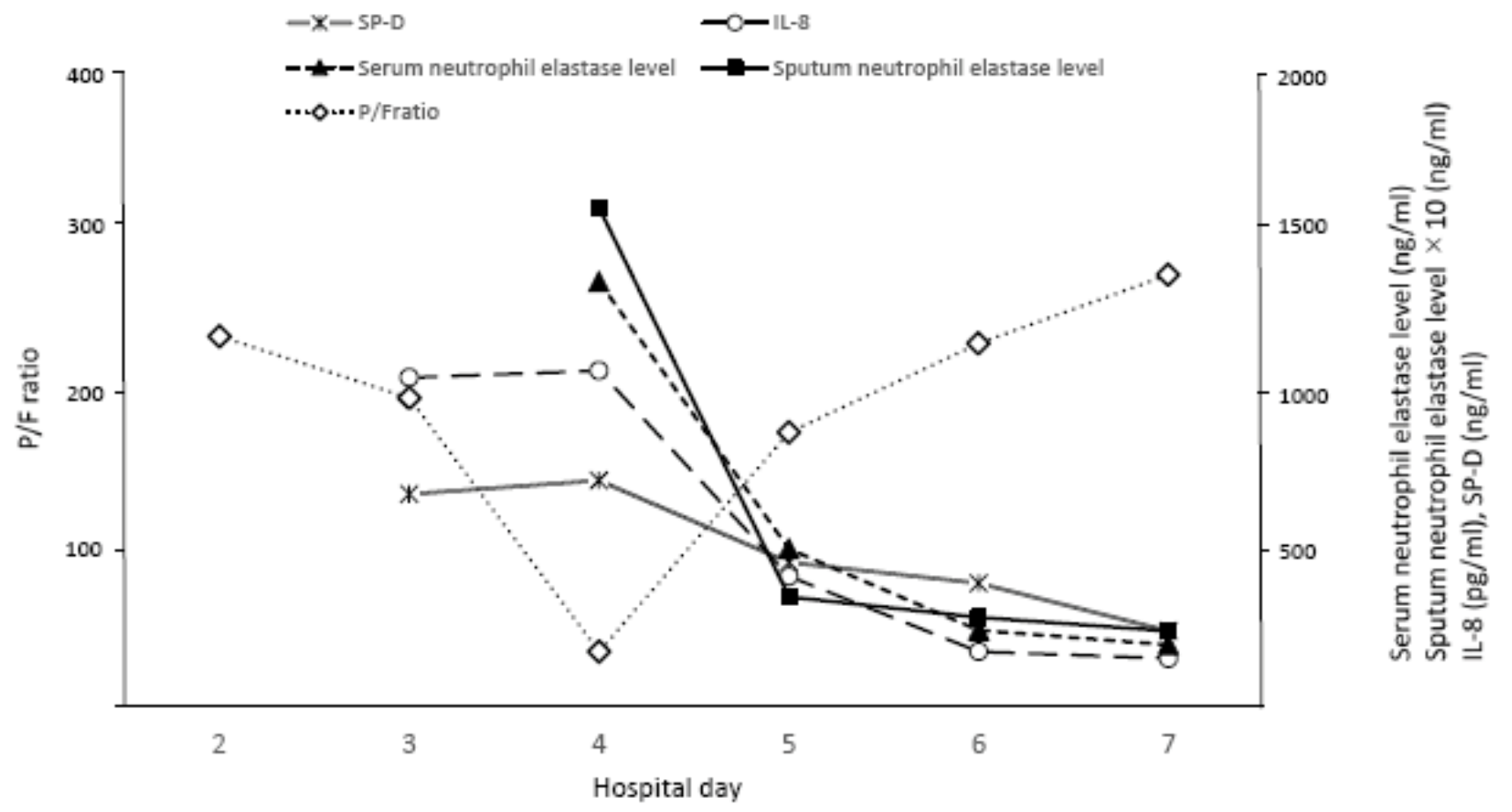

\section{IL-8: : Interleukin-8, SP-D: Surfactant protein D}

Figure 2: Blood (or sputum) levels of various humoral factors, arterial oxygen partial pressure to fractional inspired oxygen [PaO2/FiO2 (P/F)] ratio and chest $\mathrm{X}$-ray findings over time following admission.

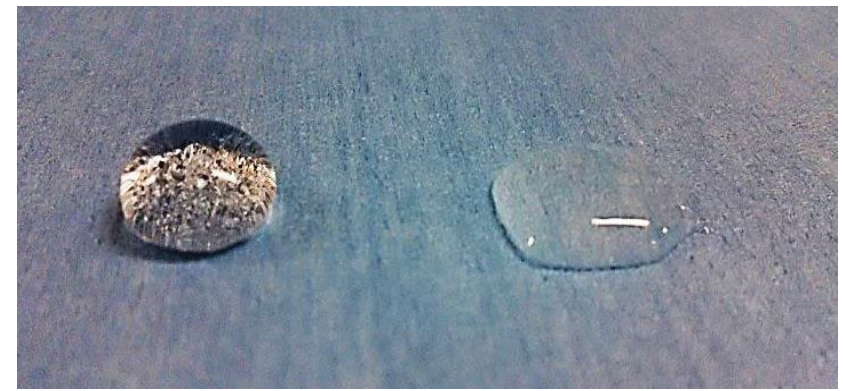

Figure 3: $200 \mu$ l of distilled water was dropped on a waterproof surgical cloth to form a sphere, and when a small amount of ethyl alcohol was dropped onto it, the sphere immediately flattened and the surface tension decreased.

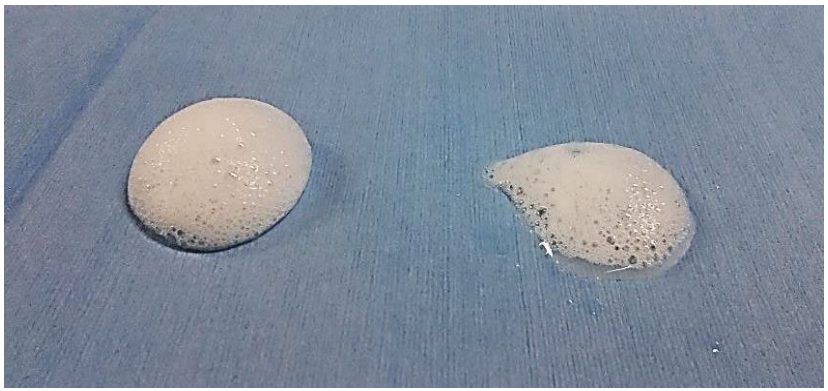

Figure 4: The foam began disappearing immediately even when a small amount of ethyl alcohol was sprayed on the foam cleanser detergent, completely disappearing in about $10 \mathrm{~s}$. 
Citation: Takahashi G, Endo S (2018) Improvement of Acute Respiratory Distress Syndrome with Ethyl Alcohol Infusion into the Airway: A Case Report. J Pulm Respir Med 8: 464. doi: 10.4172/2161-105X.1000464

the respiratory condition improved as well. Each marker markedly decreased with the improvement of $\mathrm{P} / \mathrm{F}$ ratio.

\section{Availability of Data and Material}

The data generated and analyzed in this study are included in this published article and its additional files. The original datasets used for this study are not publicly available due to the existing regulation, and only can be shared upon the approval of the directors of the corresponding hospitals.

\section{References}

1. Bernard GR, Artigas A, Brigham KL, Carlet J, Falke K, et al. (1994) The american-european consensus conference on ARDS. Definition, mechanisms, relevant outcomes and clinical trial coordination. Am J Respir Crit Care Med 149: 818-824.

2. Nakae H, Endo S, Yaegashi Y, Go W, Imai S, et al. (2004) Surfactant protein-D and polumorphonuclear leukocyte elastase concentratios in patients with septic acute respiratory distress syndrome. Crit Care \& Shock 7: 159-163.
3. Luisada AA, Goldmann MA, Weyl R (1952) Alcohol vapor by inhalation in the treatment of acute pulmonary edema. Circulation 5: 363-369.

4. Endo S, Inada K, Yamada Y, Takakuwa T, Kasai T, et al. (1994) Plasama endotoxin and cytokine Levels in patients with hemorrhagic shock. Crit Care Med 22: 949-955.

5. Endo S, Inada K, Ceska K, Takakuwa T, Yamada Y, et al. (1995) Plasma interleukin 8 and polymorphonuclear leukocyte elastase concentrations in patients with septic shock. J Inflamm 45: 136-142.

6. Nakae H, Endo S, Takakuwa T. (1995) Relationship between alpha-tocophero and polymorphonuclear leukocyte elasatase in septic adult respiratory distress syndrome. Res Commun Molecul Pathol Pharmacol 89: 93-100.

7. King RJ, Clements JA (1972) Surfactant active materials from dog. II. Composition and physiological correlations. Am J Physiol 223: 715-726.

8. Waugh WH (1993) Potential use of warm butyl alcohol vapor as adjunct agent in the emergency treatment of sea water wet near-drowing. Am J Emerg Med 11: $20-27$.

9. Boxman D (1958) Alcohol vapor in the emergency treatment of acute pulmonary edema. J Am Osteopath Assoc 57: 659-661. 